\title{
Coulomb dissociation of light nuclei
}

\author{
J. A. Tostevin, S. Rugmai, and R. C. Johnson \\ Department of Physics, School of Physical Sciences, University of Surrey, Guildford, Surrey, GU2 5XH, United Kingdom
}

(Received 27 January 1998)

\begin{abstract}
We present an alternative theoretical formulation of the Coulomb dissociation process. We apply the formalism to the deuteron as an example of a weakly bound two-body composite nucleus with a charged core and a neutral valence particle and for which exclusive experimental data exist in an appropriate kinematical regime. The theoretical scheme assumes that the projectile excitation is predominantly to states with low internal energy and is expected to be applicable at incident projectile energies of tens of $\mathrm{MeV}$ per nucleon and above. A readily calculable expression for the quantum mechanical breakup transition amplitude is obtained without the use of the distorted-wave Born approximation weak channel coupling approximation or of additional approximations for finite-range effects. Calculations are presented, analyzed, and compared with high precision, kinematically complete, measurements of elastic deuteron dissociation into very forward scattering angles. The calculations and data support the importance of the Coulomb breakup mechanism under these kinematical conditions. [S0556-2813(98)00206-4]
\end{abstract}

PACS number(s): 24.10.Eq, 24.50.+g, 25.10.+s, 25.45.-z

\section{INTRODUCTION}

The Coulomb breakup of weakly bound composite systems has been studied extensively in nuclear physics. The relationship between the cross section for Coulomb breakup and that for the inverse low-energy nuclear capture process [1] has also stimulated much experimental and theoretical effort for applications to nuclear astrophysics. These theoretical ideas and applications are discussed and reviewed in Ref. [2]. Analyses of Coulomb dissociation of the recently discovered, and very weakly bound, neutron halo nuclei [3] have also been numerous, e.g., Refs. [4,5] and references therein. Coulomb dissociation is believed to be a significant reaction channel in the scattering of such nuclei from a stable heavy target nucleus. Halo nuclei are indeed an exciting application but the low intensities of the available secondary beams of these nuclei means that experimental data are still quite limited-although they are improving rapidly.

In this paper we reconsider calculations of the Coulomb dissociation of a weakly bound two-body composite nucleus, consisting of a charged core and a neutral valence particle, by a charged target at energies of a few tens of $\mathrm{MeV}$ per nucleon and above. Within the framework of this three-body model description we present a theory which makes two approximations - that the dominant projectile breakup configurations excited are in the low-energy continuum and that the valence particle does not interact with the target. The theory is fully quantum mechanical and is also nonperturbative. It will be shown to lead to a factored expression for the quantum mechanical breakup transition amplitude similar to forms reported previously, but which were derived using approximations to distorted-wave Born approximation (DWBA) theory. The result of the present approach differs significantly from these in both detail and in interpretation. We show that, without the use of DWBA weak coupling approximations or of additional approximations, we are able to calculate explicitly the quantum mechanical breakup transition amplitude and so make comparisons with the most exclusive experimental data available.
The theory presented here offers, in certain cases, an alternative scheme to semiclassical descriptions of the excitation process in the appropriate energy regime. Such semiclassical approaches are of many forms, but most recently these have developed along the lines of coupled channels, e.g., Ref. [4], and direct solution of the time-dependent equation, e.g., Ref. [5], and are usually based upon a truncated multipole expansion of the inter-nuclear electromagnetic interaction. In the present analysis there is no such restriction to Coulomb multipole excitations. In addition, since our approach is nonperturbative, there is no truncation made in the order of excitation corrections. As will be seen, we require no zero-range approximations to interactions [6,7], and we make no reference to, or make approximations of, assumed semiclassical trajectories.

A crucial requirement for our analysis, however, is that the Coulomb interaction due to the target charge acts only on a single charged fragment in the composite few-body projectile. The method is therefore not immediately applicable to many very interesting situations. These include the Coulomb dissociation of ${ }^{8} \mathrm{~B}$, for which semiclassical methods have been applied extensively to consider higher order excitation and multipole effects [8].

For a deuteron projectile, and unlike halo nuclei, there are no ambiguities in the theoretical analysis associated with the projectile's structure. The theory is therefore first compared here with precise, kinematically complete, deuteron elastic breakup data, measured in a restricted solid angles geometry at very forward angles [9-11]. Strong indications of the importance of dipole Coulomb breakup for such a restricted detection geometry have been reported previously in the (d,pn) reaction at $E_{d}=56 \mathrm{MeV}$ [11]. This dipole dominance was manifest empirically as a very characteristic double peaking in the measured triple differential cross section as a function of detected proton laboratory energy. This dependence was reproduced qualitatively, but not quantitatively, in Ref. [11] from a consideration of the post and prior form DWBA amplitudes for Coulomb breakup. We reconsider the analysis of these $E_{d}=56 \mathrm{MeV}$ data and also subsequent data 


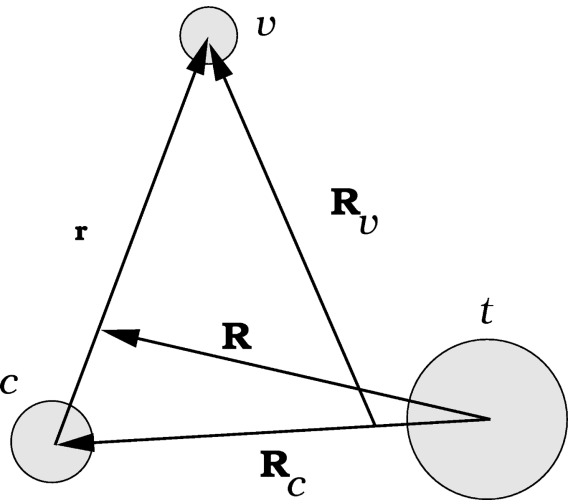

FIG. 1. Definition of the coordinate system adopted for the core, valence, and target three-body systems.

at the higher incident deuteron energies of 140 and 270 $\mathrm{MeV}$.

The method detailed here generalizes transparently to the breakup of those halo nuclei with a single charged constituent, such as ${ }^{11} \mathrm{Li},{ }^{11} \mathrm{Be}$, and ${ }^{19} \mathrm{C}$. Applications to the Coulomb breakup of such one- and two-neutron halo nuclei will be discussed elsewhere.

\section{FORMALISM}

We consider the Coulomb interaction induced dissociation of a bound two-body projectile nucleus $(p)$ by a target nucleus $(t)$, of mass $m_{t}$ and charge $Z_{t}$. The projectile ground state is assumed to be a bound state $\phi_{0}$ of a charged core (c), of mass $m_{c}$ and charge $Z_{c}$, and a neutral valence particle $(v)$ of mass $m_{v}$. In the case of the deuteron then of course the projectile has only one bound state. In the present analysis we neglect strong interaction induced breakup effects. We therefore assume that the projectile interacts with the target only through a (Coulomb) interaction $V_{c t}$ between the core particle and the target nucleus. It is assumed, and is vital for the solution used in the subsequent analysis, that the valence particle-target interaction $V_{v t}$ can be neglected (or that $\left.V_{c t} \gg V_{v t}\right)$. We adopt the system of coordinates shown in Fig. 1.

The Schrödinger equation satisfied by the scattering wave function of this three-body $(c+v+t)$ system $\Psi_{\mathbf{q}_{p}}^{(+)}(\mathbf{r}, \mathbf{R})$ when the projectile is incident with momentum $\mathbf{q}_{p}$ in the center of mass (c.m.) frame, is therefore

$$
\left[T_{\mathbf{R}}+V_{c t}\left(\mathbf{R}-\gamma_{v c} \mathbf{r}\right)+H_{v c}-E\right] \Psi_{\mathbf{q}_{p}}^{(+)}(\mathbf{r}, \mathbf{R})=0 .
$$

Here $H_{v c}=T_{\mathbf{r}}+V_{v c}(\mathbf{r})$ is the internal Hamiltonian for the valence and core particle system with relative coordinate $\mathbf{r}$, $T_{\mathbf{r}}$ is the relative motion kinetic energy operator, and $V_{v c}$ is their binding potential. The vector $\mathbf{R}$ is the projectile-target separation and $T_{\mathbf{R}}$ is the corresponding kinetic energy operator. The quantity $\gamma_{v c}=m_{v} /\left(m_{v}+m_{c}\right)$, so $\mathbf{R}-\gamma_{v c} \mathbf{r}=\mathbf{R}_{c}$ is the target-core separation. The projectile ground state wave function $\phi_{0}(\mathbf{r})$ satisfies

$$
H_{v c} \phi_{0}(\mathbf{r})=-\varepsilon_{0} \phi_{0}(\mathbf{r}) .
$$

The required three-body wave function $\Psi_{\mathbf{q}_{p}}^{(+)}(\mathbf{r}, \mathbf{R})$ therefore satisfies the scattering boundary conditions

$$
\Psi_{\mathbf{q}_{p}}^{(+)}(\mathbf{r}, \mathbf{R})=e^{i \mathbf{q}_{p} \cdot \mathbf{R}} \phi_{0}(\mathbf{r})+\text { outgoing waves, }
$$

where, for a projectile with a single bound state, the outgoing waves include only elastic scattering and elastic breakup channels. More generally, the outgoing waves will also include terms from any inelastically excited bound states. The incident plane wave boundary condition stated in Eq. (3) is of course strictly incorrect in the presence of unscreened Coulomb interactions. Our final formulas can be justified, however, as is usual, by considering the limit of the appropriately screened Coulomb problem.

\section{A. Adiabatic approximation}

An essential step in any discussion of an adiabatic approximation is that two sets of dynamical variables must be defined. One set is then identified as a high-energy (and fast) set and the other as a low-energy (and slow) set. In the present context we identify the energetic or fast variable with $\mathbf{R}$, the projectile's center of mass translational motion, and the slow variable with $\mathbf{r}$, its internal motion. At high incident energies (and large $q_{p}$ ), and for an extended $\phi_{0}$, this division is natural given the form of the entrance channel boundary condition in Eq. (3). We assume therefore that the valencecore relative excitation energies $\varepsilon$, associated with those broken-up configurations which are strongly coupled in the three-body Schrödinger equation, Eq. (1), are such that $\varepsilon$ $\ll E$, where $E$ is the incident energy of the projectile.

The actual amplitudes with which the spectrum of breakup states of $H_{v c}$ are excited in the collision will of course be dictated by the strengths and geometries of the tidal forces experienced by the projectile's constituents. In the present case these are only $V_{c t}$, the Coulomb interaction between the core and target. The long range of this interaction means that it can act repeatedly, to high order, but, because of its slow spatial variation, matrix elements of $V_{c t}$ will only couple states of $H_{v c}$ in close proximity in relative energy.

Assuming $\varepsilon \ll E$, little error will be expected upon replacing $H_{v c}$ in Eq. (1) by a representative constant energy. Moreover, if this constant is chosen as $-\varepsilon_{0}$, we also guarantee that the solution of the resulting approximate three-body equation satisfies the correct incident wave boundary conditions and that the dominant elastic channel component in the wave function has the correct channel energy. In this physical (adiabatic) limit, the three-body equation therefore reads

$$
\left[-\frac{\hbar^{2}}{2 \mu_{p}} \nabla_{\mathbf{R}}^{2}+V_{c t}\left(\mathbf{R}-\gamma_{v c} \mathbf{r}\right)-E_{0}\right] \bar{\Psi}_{\mathbf{q}_{p}}^{(+)}(\mathbf{r}, \mathbf{R})=0,
$$

where $E_{0}=E+\varepsilon_{0}=\hbar^{2} q_{p}^{2} / 2 \mu_{p}$ is the incident c.m. kinetic energy and $\mu_{p}=m_{p} m_{t} /\left(m_{p}+m_{t}\right)$ is the projectile-target reduced mass.

The approximation involved here, replacing $H_{v c}$ by $-\varepsilon_{0}$, is selectively referred to as either an adiabatic approximation [12], in the few-body reactions theory literature, or, more usually in the Coulomb excitation literature, as a sudden approximation [2]. We adopt the former usage. The approximation is seen to assume, because of the low excitation energies involved, that it is a good approximation to treat the full excitation spectrum of $H_{v c}$ as being degenerate in energy 
with the ground state. The possible shortcomings of the approximate three-body wave function $\bar{\Psi}_{\mathbf{q}_{p}}^{(+)}$calculated using this scheme, in certain regions of the six-dimensional $(\mathbf{r}, \mathbf{R})$ space, are discussed in the following subsections.

\section{B. Adiabatic model wave function}

It is important for the subsequent discussion to appreciate that the dependence of the approximate three-body Schrödinger equation, Eq. (4), on the core-valence particle separation $\mathbf{r}$ is now only parametric. Thus Eq. (4) has to be solved for all values of a fixed separation $\mathbf{r}$. We show that this solution can be expressed exactly $[13,14]$ as a product of three factors.

We introduce the operator $U_{\mathbf{R}}(\mathbf{x})$, which translates the variable $\mathbf{R}$ through $-\mathbf{x}$, i.e., $U_{\mathbf{R}}(\mathbf{x})=\exp \left(-\mathbf{x} \cdot \nabla_{\mathbf{R}}\right)$. Clearly therefore the potential operator $V_{c t}$ in Eq. (4) can be expressed as

$$
V_{c t}\left(\mathbf{R}_{c}\right)=V_{c t}\left(\mathbf{R}-\gamma_{v c} \mathbf{r}\right)=U_{\mathbf{R}}\left(\gamma_{v c} \mathbf{r}\right) V_{c t}(\mathbf{R}) U_{\mathbf{R}}^{\dagger}\left(\gamma_{v c} \mathbf{r}\right),
$$

and, since $\left[U_{\mathbf{R}}\left(\gamma_{v c} \mathbf{r}\right), \nabla_{\mathbf{R}}^{2}\right]=0$, then

$$
\left[-\frac{\hbar^{2}}{2 \mu_{p}} \nabla_{\mathbf{R}}^{2}+V_{c t}(\mathbf{R})-E_{0}\right]\left[U_{\mathbf{R}}^{\dagger}\left(\gamma_{v c} \mathbf{r}\right) \bar{\Psi}_{\mathbf{q}_{p}}^{(+)}(\mathbf{r}, \mathbf{R})\right]=0 .
$$

Evidently, the most general form of the solution $U_{\mathbf{R}}^{\dagger}\left(\gamma_{v c} \mathbf{r}\right) \bar{\Psi}_{\mathbf{q}_{p}}^{(+)}(\mathbf{r}, \mathbf{R})$ of this equation is the product of (i) an arbitrary function $\mathcal{F}(\mathbf{r})$ of the core-valence particle separation, and (ii) a projectile distorted wave $\chi_{\mathbf{q}_{p}}^{(+)}(\mathbf{R})$, and which satisfies the Schrödinger equation

$$
\left[-\frac{\hbar^{2}}{2 \mu_{p}} \nabla_{\mathbf{R}}^{2}+V_{c t}(\mathbf{R})-E_{0}\right] \chi_{\mathbf{q}_{p}}^{(+)}(\mathbf{R})=0 .
$$

In the present context therefore $\chi_{\mathbf{q}_{p}}^{(+)}$is a three-dimensional Coulomb distorted wave which describes the scattering of the projectile, of mass $\mu_{p}$ and considered pointlike, by the Coulomb interaction $V_{c t}$. The required three-body solution of Eq. (4) is therefore

$$
\bar{\Psi}_{\mathbf{q}_{p}}^{(+)}(\mathbf{r}, \mathbf{R})=\mathcal{F}(\mathbf{r})\left[U_{\mathbf{R}}\left(\gamma_{v c} \mathbf{r}\right) \chi_{\mathbf{q}_{p}}^{(+)}(\mathbf{R})\right]=\mathcal{F}(\mathbf{r}) \chi_{\mathbf{q}_{p}}^{(+)}\left(\mathbf{R}_{c}\right),
$$

where $\chi_{\mathbf{q}_{p}}^{(+)}$has now to be evaluated at the core's position coordinate $\mathbf{R}_{c}$. We note that, since $\left[U_{\mathbf{R}}\left(\gamma_{v c} \mathbf{r}\right), H_{v c}\right] \neq 0$, this product solution follows from Eq. (1) only when $H_{v c}$ is treated adiabatically.

The as yet unspecified multiplicative function $\mathcal{F}(\mathbf{r})$ in Eq. (8) must now be chosen so that the particular solution satisfies the required incident wave boundary condition, Eq. (3), as well as Eq. (4). Consideration of the incident wave boundary condition satisfied by $\chi_{\mathbf{q}_{p}}^{(+)}\left(\mathbf{R}_{c}\right)$, i.e.,

$$
\chi_{\mathbf{q}_{p}}^{(+)}\left(\mathbf{R}_{c}\right)=e^{i \mathbf{q}_{p} \cdot\left(\mathbf{R}-\gamma_{v c} \mathbf{r}\right)}+\text { outgoing waves, }
$$

and by $\bar{\Psi}_{\mathbf{q}_{p}}^{(+)}(\mathbf{r}, \mathbf{R})$, Eq. (3), shows that we require that

$$
\mathcal{F}(\mathbf{r})=\exp \left(i \gamma_{v c} \mathbf{q}_{p} \cdot \mathbf{r}\right) \phi_{0}(\mathbf{r})
$$

Hence the exact solution of the adiabatic three-body problem, Eq. (4), is $[13,14]$

$$
\bar{\Psi}_{\mathbf{q}_{p}}^{(+)}(\mathbf{r}, \mathbf{R})=\exp \left(i \gamma_{v c} \mathbf{q}_{p} \cdot \mathbf{r}\right) \chi_{\mathbf{q}_{p}}^{(+)}\left(\mathbf{R}_{c}\right) \phi_{0}(\mathbf{r})
$$

It is important to stress that this three-body wave function retains breakup components. These are manifest in the extremely complex dependence of the wave function on $\mathbf{r}$, beyond that in $\phi_{0}$, entering both $\chi_{\mathbf{q}_{p}}^{(+)}\left(\mathbf{R}-\gamma_{v c} \mathbf{r}\right)$ and in the exponential factor $\exp \left(i \gamma_{v c} \mathbf{q}_{p} \cdot \mathbf{r}\right)$.

In the following we use the three-body wave function $\bar{\Psi}_{\mathbf{q}_{p}}^{(+)}$to calculate the Coulomb dissociation process. The application of this three-body model solution to the elastic scattering of halo nuclei has recently been presented elsewhere $[13,15]$. In that case the core-target interaction $V_{c t}$ was not assumed to be purely Coulomb but, since there $m_{v} / m_{c} \ll 1$, $V_{c t}$ could reasonably be assumed to be dominant, i.e., that $V_{c t} \gg V_{v t}$. A careful discussion of the validity of the adiabatic approximation in both the nuclear and Coulomb dominated situations is presented in Ref. [14].

\section{Use of the adiabatic wave function}

We have shown that Eq. (11) is the exact solution of the stated three-body model, given only the adiabatic assumption. The very explicit form of the solution in this case makes it clear that, at large core-valence particle separations $\mathbf{r} \rightarrow \infty$ the presence of the factor $\phi_{0}$ means that $\bar{\Psi}_{\mathbf{q}_{p}}^{(+)}$vanishes (at least exponentially) in this region. This region contains contributions from some parts of the breakup flux and thus it is evident that for large values of $|\mathbf{r}|$, in any direction, $\bar{\Psi}_{\mathbf{q}_{p}}^{(+)}$will be inaccurate-a consequence of our assumption that the entire spectrum of $H_{v c}$ is degenerate with the ground state.

It follows that, to use the three-body wave function of Eq. (11) to calculate a Coulomb breakup amplitude, we must consider its limitation to only certain regions of the sixdimensional $(\mathbf{r}, \mathbf{R})$ space. We therefore select the breakup matrix element to be evaluated accordingly and, in particular, we do not attempt to extract the breakup amplitude from the asymptotics of our approximate adiabatic solution. In fact we use the post-form of the exact quantum mechanical breakup amplitude.

This amplitude, from a projectile initial state $\mathbf{q}_{p}$ to a threebody final state with core momentum $\mathbf{q}_{c}$ and valence particle momentum $\mathbf{q}_{v}$ in the c.m. frame, is

$$
\begin{aligned}
T_{\mathrm{bu}}\left(\mathbf{q}_{v} \mathbf{q}_{c}, \mathbf{q}_{p}\right)= & \int d \mathbf{R} \int d \mathbf{r} e^{-i \mathbf{q}_{v} \cdot \mathbf{R}_{v}} \chi_{\mathbf{q}_{c}}^{(-) *}\left(\mathbf{R}_{c}\right) \\
& \times V_{v c}(\mathbf{r}) \Psi_{\mathbf{q}_{p}}^{(+)}(\mathbf{r}, \mathbf{R}),
\end{aligned}
$$

where $\chi_{\mathbf{q}_{c}}^{(-)}$is an in-going waves Coulomb distorted wave for the core fragment. Due to $V_{v c}$, the integrand in Eq. (12) involves only finite $\mathbf{r}$ and so does not involve the three-body wave function in regions where our approximate solution 
will be inaccurate. We therefore insert $\bar{\Psi}_{\mathbf{q}_{p}}^{(+)}$as an approximation to $\Psi_{\mathbf{q}_{p}}^{(+)}$within this post-form amplitude giving

$$
\bar{T}_{\mathrm{AD}}\left(\mathbf{q}_{v} \mathbf{q}_{c}, \mathbf{q}_{p}\right)=\left\langle e^{i \mathbf{q}_{v} \cdot \mathbf{R}_{v}} \chi_{\mathbf{q}_{c}}^{(-)}\left(\mathbf{R}_{c}\right)\left|V_{v c}(\mathbf{r})\right| \bar{\Psi}_{\mathbf{q}_{p}}^{(+)}(\mathbf{r}, \mathbf{R})\right\rangle .
$$

Our approach therefore goes beyond the lowest order adiabatic approximation as described by $\Psi_{\mathbf{q}_{p}}^{(+)}$. This can be seen as follows. We first rewrite the exact three-body Schrödinger equation of Eq. (1), prior to any adiabatic approximation, as

$$
\left[E-T_{\mathbf{R}_{v}}-T_{\mathbf{R}_{c}}-V_{c t}\left(\mathbf{R}_{c}\right)\right] \Psi_{\mathbf{q}_{p}}^{(+)}(\mathbf{r}, \mathbf{R})=V_{v c}(\mathbf{r}) \Psi_{\mathbf{q}_{p}}^{(+)}(\mathbf{r}, \mathbf{R}),
$$

where $T_{\mathbf{R}_{v}}$ and $T_{\mathbf{R}_{c}}$ are the kinetic energies in the coordinates $\mathbf{R}_{v}$ and $\mathbf{R}_{c}$ of Fig. 1 and involve the reduced masses $\mu_{v}$ $=m_{v}\left(m_{c}+m_{t}\right) /\left(m_{v}+m_{c}+m_{t}\right)$ and $\mu_{c}=m_{c} m_{t} /\left(m_{c}+m_{t}\right)$, respectively. Since now a calculation of the source term in Eq. (14) requires only finite separations $\mathbf{r}$, we can use the adiabatic wave function $\bar{\Psi}_{\mathbf{q}_{p}}^{(+)}$as a good approximation to $\Psi_{\mathbf{q}_{p}}^{(+)}$within this source term only. This yields the inhomogeneous equation

$$
\left[E-T_{\mathbf{R}_{v}}-T_{\mathbf{R}_{c}}-V_{c t}\left(\mathbf{R}_{c}\right)\right] \hat{\Psi}_{\mathbf{q}_{p}}^{(+)}(\mathbf{r}, \mathbf{R})=V_{v c}(\mathbf{r}) \bar{\Psi}_{\mathbf{q}_{p}}^{(+)}(\mathbf{r}, \mathbf{R}),
$$

and a (first) iterated approximate wave function $\hat{\Psi}_{\mathbf{q}_{p}}^{(+)}$. This equation has formal solution

$$
\begin{aligned}
\hat{\Psi}_{\mathbf{q}_{p}}^{(+)}(\mathbf{r}, \mathbf{R})= & \frac{1}{(2 \pi)^{3}} \int d \mathbf{k}_{v}^{\prime} \int d \mathbf{R}_{v}^{\prime} \int d \mathbf{R}_{c}^{\prime} \\
& \times e^{i \mathbf{k}_{v}^{\prime} \cdot\left(\mathbf{R}_{v}-\mathbf{R}_{v}^{\prime}\right)} G_{E_{c}}^{(+)}\left(\mathbf{R}_{c}, \mathbf{R}_{c}^{\prime}\right) \\
& \times V_{v c}\left(\mathbf{r}^{\prime}\right) \bar{\Psi}_{\mathbf{q}_{p}}^{(+)}\left(\mathbf{r}^{\prime}, \mathbf{R}^{\prime}\right),
\end{aligned}
$$

or, upon integrating over the directions of $\mathbf{k}_{v}^{\prime}$,

$$
\begin{aligned}
\hat{\Psi}_{\mathbf{q}_{p}}^{(+)}(\mathbf{r}, \mathbf{R})= & \frac{-i}{(2 \pi)^{2}} \int d \mathbf{R}_{v}^{\prime} \int d \mathbf{R}_{c}^{\prime} \int_{-\infty}^{\infty} k_{v}^{\prime} d k_{v}^{\prime} \\
& \times \frac{e^{i k_{v}^{\prime}\left|\mathbf{R}_{v}-\mathbf{R}_{v}^{\prime}\right|}}{\left|\mathbf{R}_{v}-\mathbf{R}_{v}^{\prime}\right|} G_{E_{c}}^{(+)}\left(\mathbf{R}_{c}, \mathbf{R}_{c}^{\prime}\right) \\
& \times V_{v c}\left(\mathbf{r}^{\prime}\right) \bar{\Psi}_{\mathbf{q}_{p}}^{(+)}\left(\mathbf{r}^{\prime}, \mathbf{R}^{\prime}\right),
\end{aligned}
$$

where $G_{E_{c}}^{(+)}$is the core-target Green's function in the presence of potential $V_{c t}$ :

$$
\begin{aligned}
& G_{E_{c}}^{(+)}\left(\mathbf{R}_{c}, \mathbf{R}_{c}^{\prime}\right)=\left\langle\mathbf{R}_{c}\left|\left[E_{c}^{+}-T_{\mathbf{R}_{c}}-V_{c t}\right]^{-1}\right| \mathbf{R}_{c}^{\prime}\right\rangle, \\
& E_{c}=E-\hbar^{2} k_{v}^{\prime 2} / 2 \mu_{v} .
\end{aligned}
$$

Using the asymptotic behavior of the two Green's functions in the integrand as $\mathbf{R}_{v}$ and $\mathbf{R}_{c} \rightarrow \infty$ in the directions $\mathbf{R}_{c}$ $\rightarrow R_{c} \hat{\mathbf{q}}_{c}$ and $\mathbf{R}_{v} \rightarrow R_{v} \hat{\mathbf{q}}_{v}$, the final integral over $k_{v}^{\prime}$ can be carried out using the stationary-phase point of $k_{v}^{\prime} R_{v}+k_{c} R_{c}$ [16]. This derives the expected three-body asymptotics [17] and the approximate transition amplitude $\bar{T}_{\mathrm{AD}}$ of Eq. (13), i.e.,

$$
\begin{aligned}
\hat{\Psi}_{\mathbf{q}_{p}}^{(+)}(\mathbf{r}, \mathbf{R}) \rightarrow & {[\text { factors }] \times \frac{e^{i\left(q_{v} R_{v}+q_{c} R_{c}\right)}}{\mathcal{R}^{5 / 2}} } \\
& \times\left\langle e^{i \mathbf{q}_{v} \cdot \mathbf{R}_{v}^{\prime}} \chi_{\mathbf{q}_{c}}^{(-)}\left(\mathbf{R}_{c}^{\prime}\right)\left|V_{v c}\left(\mathbf{r}^{\prime}\right)\right| \bar{\Psi}_{\mathbf{q}_{p}}^{(+)}\left(\mathbf{r}^{\prime}, \mathbf{R}^{\prime}\right)\right\rangle,
\end{aligned}
$$

where $\mathcal{R}$ is the hyperradial variable $\mathcal{R}^{2}=\left(\mu_{v} R_{v}^{2}\right.$ $\left.+\mu_{c} R_{c}^{2}\right) / \sqrt{\mu_{v} \mu_{c}}$ and $q_{v}$ and $q_{c}$ are such as to satisfy energy conservation $\hbar^{2} q_{v}^{2} / 2 \mu_{v}+\hbar^{2} q_{c}^{2} / 2 \mu_{c}=E$. The phase space factors entering Eq. (19) are discussed in Ref. [17] and those required for the calculated cross sections are presented in the results section of this paper.

An evaluation of the breakup amplitude $\bar{T}_{\mathrm{AD}}$ of Eq. (13) is therefore formally equivalent to the solution of Eq. (15). So, although the adiabatic approximation neglects the projectile excitation energy in the calculation of the adiabatic threebody wave function $\bar{\Psi}_{\mathbf{q}_{p}}^{(+)}$, this does not mean we calculate the breakup using the $\xi=0$ approximation, the zero adiabaticity parameter limit, of semiclassical theories [2]. As the analysis above shows, our calculation of $\bar{T}_{\mathrm{AD}}$ includes correctly the final state wave functions, kinematics, and excitation energies, unlike analogous $\xi=0$ semiclassical calculations.

\section{Breakup transition amplitude}

Our approximation $\bar{T}_{\mathrm{AD}}$ to the Coulomb breakup transition amplitude is therefore

$$
\begin{aligned}
\bar{T}_{\mathrm{AD}}\left(\mathbf{q}_{v} \mathbf{q}_{c}, \mathbf{q}_{p}\right)= & \int d \mathbf{R} \int d \mathbf{r} e^{-i \mathbf{q}_{v} \cdot \mathbf{R}_{v}} \chi_{\mathbf{q}_{c}}^{(-) *}\left(\mathbf{R}_{c}\right) V_{v c}(\mathbf{r}) \\
& \times\left[e^{i \gamma_{v c} \mathbf{q}_{p} \cdot \mathbf{r}} \chi_{\mathbf{q}_{p}}^{(+)}\left(\mathbf{R}_{c}\right) \phi_{0}(\mathbf{r})\right] .
\end{aligned}
$$

Upon making a change of integration variable from $\mathbf{R}$ to $\mathbf{R}_{c}$, and noting that $\mathbf{R}_{v}=\gamma_{t c} \mathbf{R}_{c}+\mathbf{r}$, where $\gamma_{t c}=m_{t} /\left(m_{c}+m_{t}\right)$, our breakup amplitude is seen to factorize exactly as

$$
\begin{aligned}
\bar{T}_{\mathrm{AD}}\left(\mathbf{q}_{v} \mathbf{q}_{c}, \mathbf{q}_{p}\right)= & {\left[\int d \mathbf{r} e^{-i \mathbf{P}_{v} \cdot \mathbf{r}} V_{v c}(\mathbf{r}) \phi_{0}(\mathbf{r})\right] } \\
& \times\left[\int d \mathbf{R}_{c} e^{-i \mathbf{Q}_{v} \cdot \mathbf{R}_{c}} \chi_{\mathbf{q}_{c}}^{(-) *}\left(\mathbf{R}_{c}\right) \chi_{\mathbf{q}_{p}}^{(+)}\left(\mathbf{R}_{c}\right)\right] \\
= & \left\langle\mathbf{P}_{v}\left|V_{v c}\right| \phi_{0}\right\rangle\left\langle\mathbf{Q}_{v}, \chi_{\mathbf{q}_{c}}^{(-)} \mid \chi_{\mathbf{q}_{p}}^{(+)}\right\rangle,
\end{aligned}
$$

where we have defined $\mathbf{P}_{v}=\mathbf{q}_{v}-\gamma_{v c} \mathbf{q}_{p}$ and $\mathbf{Q}_{v}=\gamma_{t c} \mathbf{q}_{v}$.

The two factors in Eq. (21) delineate the structure and dynamical parts of the calculation. The overlap of the three continuum functions which arises here, $\left\langle\mathbf{Q}_{v}, \chi_{\mathbf{q}_{c}}^{(-)} \mid \chi_{\mathbf{q}_{p}}^{(+)}\right\rangle$, has been evaluated in closed form and is expressed in terms of the bremsstrahlung integral, e.g., Refs. [18,19]. This factor now contains all the dynamics of the breakup process and is readily calculated. 
The projectile structure enters through the vertex function $\left\langle\mathbf{P}_{v}\left|V_{v c}\right| \phi_{0}\right\rangle$ and is also simply evaluated given any structure model for the projectile. In Coulomb dissociation momentum can be transferred to the valence particle only by virtue of its interaction $V_{v c}$ with the core. Since the term $\gamma_{v c} \mathbf{q}_{p}$ in $\mathbf{P}_{v}$ is the fraction of the incident momentum of the projectile which is carried by the valence particle, this structure vertex displays explicitly this momentum transfer from the ground state via $V_{v c}$.

Therefore, without any approximation additional to the adiabatic assumption, Eq. (21) encompasses a fully finiterange treatment of the core-valence particle interaction $V_{v c}$. Our amplitude is thus applicable to projectiles with any ground state orbital angular momentum structure, and also includes breakup contributions from all contributing Coulomb multipoles and relative orbital angular momenta between the valence and core fragments. This amplitude clearly also differs significantly from those of DWBA theories since it includes the initial and final state interactions $V_{c t}$ and $V_{v c}$ to all orders. In the following we contrast our result with those published previously, based on approximations to the weak coupling DWBA theory.

\section{RELATIONSHIP TO DWBA APPROACHES}

Expressions with a factored structure similar to that of our Eq. (21), and which also use the bremsstrahlung integral to treat the reaction dynamics, have been presented previously, e.g., Refs. [18,20]. These alternative expressions were obtained, however, not as above, but by starting from the post form of the distorted-wave Born approximation to the breakup transition amplitude

$$
\begin{aligned}
T_{\mathrm{DW}}\left(\mathbf{q}_{v} \mathbf{q}_{c}, \mathbf{q}_{p}\right)= & \int d \mathbf{R} \int d \mathbf{r} e^{-i \mathbf{q}_{v} \cdot \mathbf{R}_{v}} \chi_{\mathbf{q}_{c}}^{(-) *}\left(\mathbf{R}_{c}\right) \\
& \times V_{v c}(\mathbf{r})\left[\chi_{\mathbf{q}_{p}}^{(+)}(\mathbf{R}) \phi_{0}(\mathbf{r})\right],
\end{aligned}
$$

and then making different additional approximations. $T_{\mathrm{DW}}$ is obtained by neglecting completely the effects of the Coulomb polarization (breakup) potential $\Delta V(\mathbf{r}, \mathbf{R})=V_{c t}\left(\mathbf{R}_{c}\right)$ $-V_{c t}(\mathbf{R})$ in the calculation of the three-body wave function. It thus replaces $\Psi_{\mathbf{q}_{p}}^{(+)}(\mathbf{r}, \mathbf{R})$ by

$$
\Psi_{\mathbf{q}_{p}}^{(+)}(\mathbf{r}, \mathbf{R}) \approx \chi_{\mathbf{q}_{p}}^{(+)}(\mathbf{R}) \phi_{0}(\mathbf{r})
$$

in Eq. (12), where $\chi_{\mathbf{q}_{p}}^{(+)}(\mathbf{R})$ is the entrance channel Coulomb distorted wave (for an assumed point projectile), the argument of which is the projectile c.m. coordinate. It is assumed in writing Eq. (22) that breakup channels are very weakly coupled and hence that this coupling need only be treated to first order. We reiterate that the adiabatic formulation leading to Eq. (21) did not use such Born approximation considerations at any stage.

For a realistic and hence finite ranged $V_{v c}$ the DWBA amplitude $T_{\text {DW }}$ has itself not been calculated exactly and further approximations are therefore applied-involving approximate treatments of, or the complete neglect of, these finite-range effects. In Coulomb breakup, however, the fact that the Coulomb interaction acts at the charged core and not at the center of mass of the projectile is absolutely critical and therefore requires a very careful consideration of the deviations of $\mathbf{R}_{c}$ from $\mathbf{R}$. Our adiabatic model formulation retained these finite $\mathbf{r}$ effects explicitly. A key result is that, in Eq. (21), the projectile vertex appears evaluated at $\mathbf{P}_{v}$ $=\mathbf{q}_{v}-\gamma_{v c} \mathbf{q}_{p}$, where $\mathbf{q}_{v}$ and $\mathbf{q}_{p}$ are the outgoing valence particle and incident projectile asymptotic momenta, and hence $\mathbf{P}_{v}$ is the momentum transferred to the neutron in the breakup.

In the following we examine critically those approximations to $T_{\mathrm{DW}}$ previously used in the literature which lead to expressions similar to our Eq. (21). We carry out this discussion with the deuteron dissociation process in mind, for incident energies in the range $30-140 \mathrm{MeV}$ per nucleon. Since in the $(d, p n)$ reaction $V_{v c}$ is the free $n p$ interaction, $T_{\mathrm{DW}}$ in this case presents the physical situation in which the range $\mathcal{R}_{v c}$ of the transition interaction is the smallest encountered. In addition the $(d, p n)$ reaction involves the least massive physical projectile and dissociated fragments. Thus, for a reaction of a given incident energy per nucleon, the wave functions entering the DWBA matrix element will have longer wavelengths than in the analogous reactions with heavier projectiles. It follows that the ratio of the interaction range $\mathcal{R}_{v c}$ to the wavelengths $\lambda$ of the distorted waves entering Eq. (22) is at its smallest for the $(d, p n)$ reaction. This ratio is a critical measure for assessing the likely importance of retaining finite-range effects within the breakup amplitude.

\section{A. Method of zero-range DWBA}

For the $(d, p n)$ reaction, $V_{v c}$ is the physical $n p$ interaction with a (finite) range $\mathcal{R}_{v c} \approx 1.4 \mathrm{fm}$. For a zero-range approximation to the DWBA matrix element, Eq. (22), to provide a reasonable quantitative estimate of the amplitude requires that all functions (other than $V_{v c} \phi_{0}$ ) appearing in the integrand have characteristic lengths $\lambda$, for a significant functional variation, such that $\lambda \gg \mathcal{R}_{v c}$. When this is the case, as at very low (tandem) energies, then the zero-range approximation neglects variations of the distorted waves product $e^{-i \mathbf{q}_{v} \cdot \mathbf{R}_{v}} \chi_{\mathbf{q}_{c}}^{(-) *}\left(\mathbf{R}_{c}\right) \chi_{\mathbf{q}_{p}}^{(+)}(\mathbf{R})$ for those $|\mathbf{r}| \leqslant \mathcal{R}_{v c}$.

However, even in the case of the light-ion $(d, p n)$ reaction, at say $100 \mathrm{MeV}$, the wave number of the incident deuteron is larger than $3 \mathrm{fm}^{-1}$ and thus the $\lambda$ associated with the distorted waves are comparable to $\mathcal{R}_{v c}$. The approximation that the distorted waves are constant over the range of $V_{v c} \phi_{0}$ in Eq. (22) is therefore untenable and finite-range effects are expected to be significant. This situation will be even worse for heavier systems, such as halo nuclei, where the $\mathcal{R}_{v c}$ are larger, the nuclei more weakly bound, and the projectile and fragment $\lambda$ (at the same incident energy per nucleon) are even smaller. The zero-range approximation to the post-form DWBA breakup matrix element is therefore difficult to justify physically, either in the present light-ion context or for related halo systems with similar incident energies/nucleon. This zero-range DWBA approach, and the underlying physical picture it suggests of the breakup process, particularly for higher energies or massive particles, is therefore misleading.

If nevertheless one assumes that the zero-range approximation is valid then, taking the small $\mathcal{R}_{v c}$ limit, i.e., $\mathbf{R}$ $\rightarrow \mathbf{R}_{c}$, and $\mathbf{R}_{v} \rightarrow \gamma_{t c} \mathbf{R}_{c}$, in the distorted waves integrand $e^{-i \mathbf{q}_{v} \cdot \mathbf{R}_{v}} \chi_{\mathbf{q}_{c}}^{(-) *}\left(\mathbf{R}_{c}\right) \chi_{\mathbf{q}_{p}}^{(+)}(\mathbf{R})$, Eq. (22) reduces to 


$$
T_{\mathrm{DW}}\left(\mathbf{q}_{v} \mathbf{q}_{c}, \mathbf{q}_{p}\right) \approx T_{\mathrm{ZR}}\left(\mathbf{q}_{v} \mathbf{q}_{c}, \mathbf{q}_{p}\right)=D_{0}\left\langle\mathbf{Q}_{v}, \chi_{\mathbf{q}_{c}}^{(-)} \mid \chi_{\mathbf{q}_{p}}^{(+)}\right\rangle,
$$

where

$$
D_{0}=\int d \mathbf{r} V_{v c}(\mathbf{r}) \phi_{0}(\mathbf{r})=\left\langle\mathbf{0}\left|V_{v c}\right| \phi_{0}\right\rangle
$$

Thus, when applicable, the zero-range approximation to the DWBA leads to an expression with the structure of our Eq. (21), but where the projectile vertex is evaluated at zero valence-core relative momentum-the usual zero-range strength constant $D_{0}$.

Comparison with our Eq. (21) shows that this zero-range expression is, fortuitously, equal to the $\mathbf{P}_{v}=0$ approximation to our finite-range adiabatic breakup amplitude. Since in Eq. (21) the projectile vertex appears evaluated at $\mathbf{P}_{v}=\mathbf{q}_{v}$ $-\gamma_{v c} \mathbf{q}_{p}$, the presence of $\mathbf{q}_{p}$ results in $\left|\mathbf{P}_{v}\right|$, the momentum transfer, taking on small values. As a result only relatively low momentum components of the vertex are probed in our Eq. (21). Consequently, although the application of the zerorange approximation to Eq. (22), which leads to Eq. (24), is not physically justified at the energies of interest here, the $\mathbf{P}_{v}=0$ approximation to Eq. (21) is expected to be rather good. In the deuteron breakup calculations which follow $\left|\mathbf{P}_{v}\right| \approx 0.1 \mathrm{fm}^{-1}$ at the cross section maxima. It follows that, starting from our Eq. (21), a good first approximation is to replace the vertex function by its $\mathbf{P}_{v}=0$ strength constant, although there is no reason to do so.

We summarize that the expression given on the right-hand side of Eq. (24) is, fortuitously, a rather good approximation to $\bar{T}_{\mathrm{AD}}$ of Eqs. (20) and (21). It is, however, expected to be a very poor approximation to the finite-range DWBA amplitude $T_{\mathrm{DW}}$. This is, we believe, the reason for the apparent success of such supposedly "zero-range calculations," based on Eq. (24), in comparisons with experimental data, e.g., Ref. [20].

\section{B. Method of Baur and Trautmann}

An alternative approximation scheme which leads to an amplitude of the form of Eq. (21), was introduced by Baur and Trautmann [18]. The original motivation of these authors was the study of deuteron breakup at sub-Coulomb barrier energies where the present adiabatic model is almost certainly inappropriate. Their basic method has, however, been cited in other contexts.

Their ansatz is to replace the projectile c.m. coordinate in the projectile distorted wave, in Eq. (22), by the core coordinate, i.e., $\chi_{\mathbf{q}_{p}}^{(+)}(\mathbf{R}) \rightarrow \chi_{\mathbf{q}_{p}}^{(+)}\left(\mathbf{R}_{c}\right)$. This means their approximation to the three-body wave function is

$$
\Psi_{\mathbf{q}_{p}}^{(+)}(\mathbf{r}, \mathbf{R}) \approx \chi_{\mathbf{q}_{p}}^{(+)}\left(\mathbf{R}_{c}\right) \phi_{0}(\mathbf{r}),
$$

and was argued on the basis that the Coulomb polarization potential acts to push the proton coordinate, on average, to larger radii. Since the exponential factor $\exp \left(i \gamma_{v c} \mathbf{q}_{p} \cdot \mathbf{r}\right)$ which arose in our Eq. (11) for the three-body wave function is absent, it follows that in this case,

$$
T_{\mathrm{BT}}\left(\mathbf{q}_{v} \mathbf{q}_{c}, \mathbf{q}_{p}\right)=\left\langle\mathbf{q}_{v}\left|V_{v c}\right| \phi_{0}\right\rangle\left\langle\mathbf{Q}_{v}, \chi_{\mathbf{q}_{c}}^{(-)} \mid \chi_{\mathbf{q}_{p}}^{(+)}\right\rangle .
$$

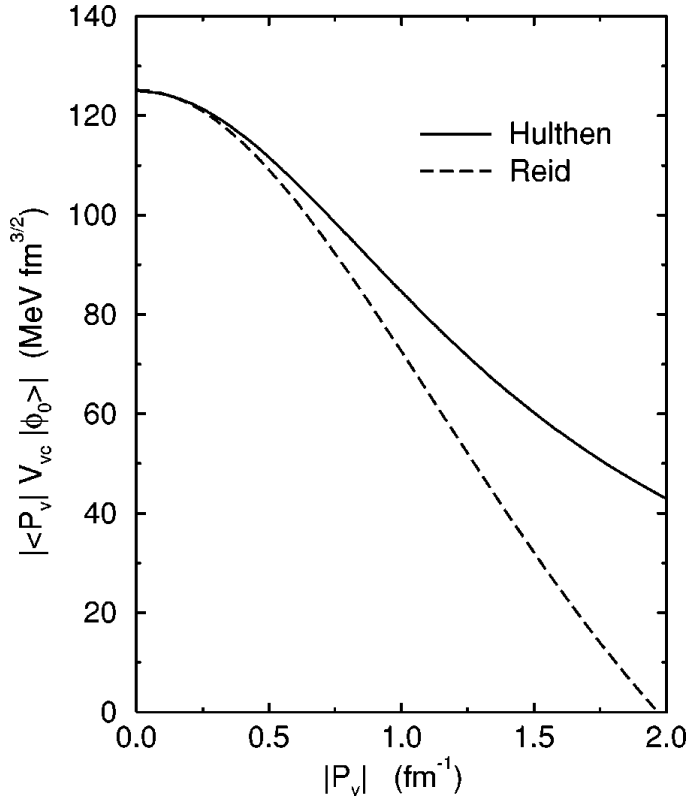

FIG. 2. Modulus of the vertex function calculated for the Hulthén and Reid soft core deuteron wave functions, as a function of the $n p$ relative momentum.

Once again this looks similar to our Eq. (21) but with the very important difference that the first term, the projectile vertex, is now evaluated at the valence particle momentum $\mathbf{q}_{v}$ in the final state - not at the momentum transfer $\mathbf{P}_{v}$ as appears in Eq. (21). While for the original application envisaged in [18], dissociation at sub-Coulomb barrier energies, the important $\left|\mathbf{q}_{v}\right|$ are small, and so $\left\langle\mathbf{q}_{v}\left|V_{v c}\right| \phi_{0}\right\rangle \approx D_{0}$, this is not the case for the applications involved in Refs. [20,21] and elsewhere.

At the energies of interest here, the relevant momenta $\mathbf{q}_{v}$ are large. They are typically in the range $1.0-2.0 \mathrm{fm}^{-1}$ in the deuteron breakup calculations of the following section. Figure 2 shows the modulus of the vertex function calculated for the Hulthén [22] and Reid soft core [23] deuteron wave functions, as a function of the $n p$ relative momentum. At momenta of $1.0-2.0 \mathrm{fm}^{-1}$ the function values for such realistic deuteron wave functions are already markedly different from their low momentum $\mathbf{q}_{v} \approx 0$ values. This difference of $\left\langle\mathbf{q}_{v}\left|V_{v c}\right| \phi_{0}\right\rangle$ from the zero-range vertex $D_{0}=\left\langle\mathbf{0}\left|V_{v c}\right| \phi_{0}\right\rangle$ will be even more acute in systems, such as halo nuclei, with reduced binding and larger $\mathcal{R}_{v c}$.

The Baur-Trautmann approximation thus yields a breakup amplitude which differs from the adiabatic theory by a factor $\left\langle\mathbf{q}_{v}\left|V_{v c}\right| \phi_{0}\right\rangle /\left\langle\mathbf{P}_{v}\left|V_{v c}\right| \phi_{0}\right\rangle$ which is dependent on the kinematics of the reaction and the observables calculated. We show the result of using this approach in the following section.

We conclude, however, that, even in deuteron induced reactions with final state valence particle momenta $q_{v}$ as small as $1 \mathrm{fm}^{-1}$, the approximate breakup amplitude $T_{\mathrm{BT}}$ of Eq. (27) will differ markedly from both $\bar{T}_{\mathrm{AD}}$ of Eq. (21) and $T_{\mathrm{ZR}}$ of Eq. (24). In particular, for any realistic $V_{v c} \phi_{0}$, it is not a reasonable approximation to replace $\left\langle\mathbf{q}_{v}\left|V_{v c}\right| \phi_{0}\right\rangle$ by $D_{0}$, which restores the calculation to zero-range form, as has been done in the literature for both deuteron and halo nucleus systems, e.g., Refs. [20,21]. 


\section{RESULTS AND ANALYSIS}

In the following we compare the predictions of calculations made using Eq. (21) with high precision measurements of the triple differential cross sections $d^{3} \sigma / d \Omega_{n} d \Omega_{p} d E_{p}$ for deuteron dissociation into very forward angles. In addition to their precision, these deuteron data are free from structure ambiguities associated with the projectile vertex. We adopt the finite-range Hulthén vertex presented explicitly in Ref. [18], and shown also in Fig. 2. Given this input the theoretical calculations we present are entirely parameter free. In the cross section formulas we continue to refer to the core and valence particles for generality. Of course $c$ is now the proton, $v$ is the neutron, and the projectile $p$ is the deuteron.

The necessary three-body final state kinematics and phase space formulas for the calculation of $d^{3} \sigma / d \Omega_{v} d \Omega_{c} d E_{c}$ are presented in Ref. [24] and also discussed in Ref. [25]. These give

$$
\frac{d^{3} \sigma}{d \Omega_{c} d \Omega_{v} d E_{c}}=\frac{2 \pi \mu_{p}}{\hbar^{2} q_{p}}\left|T\left(\mathbf{q}_{v} \mathbf{q}_{c}, \mathbf{q}_{p}\right)\right|^{2} \rho\left(E_{c}, \Omega_{c}, \Omega_{v}\right),
$$

where $\rho\left(E_{c}, \Omega_{c}, \Omega_{v}\right)$ is the density of states, per unit core particle energy interval, for detection at solid angles $\Omega_{v}$ and $\Omega_{c}$, and is

$$
\begin{aligned}
\rho\left(E_{c}, \Omega_{c}, \Omega_{v}\right)= & \frac{m_{c} m_{v} \hbar k_{c} \hbar k_{v}}{(2 \pi \hbar)^{6}} \\
& \times\left[\frac{m_{t}}{m_{v}+m_{t}+m_{v}\left(\mathbf{k}_{c}-\mathbf{K}\right) \cdot \mathbf{k}_{v} / k_{v}^{2}}\right] .
\end{aligned}
$$

Here $\hbar \mathbf{k}_{c}$ and $\hbar \mathbf{k}_{v}$ are the proton and neutron momenta in the final state and $\hbar \mathbf{K}$ the total momentum, all in the frame (c.m. or laboratory) of interest. The experimental data considered here are presented in the laboratory frame.

As will be seen in the following, the angular dependences of these calculated triple differential cross sections near zero degrees are extremely rapid and qualitatively different for light and heavy targets, and at different incident energies. It is therefore essential to integrate and then average the theoretical angular distributions over the specified experimental solid angle acceptances before final comparisons are made with data. That is, we evaluate

$$
\frac{d^{3} \sigma(\exp )}{d \Omega_{c} d \Omega_{v} d E_{c}}=\frac{1}{\Delta \Omega_{c} \Delta \Omega_{v}} \int_{\Delta \Omega_{c}, \Delta \Omega_{v}} d \Omega_{c} d \Omega_{v} \frac{d^{3} \sigma}{d \Omega_{c} d \Omega_{v} d E_{c}},
$$

where the integrations over the proton $\Delta \Omega_{c}$ and neutron $\Delta \Omega_{v}$ detection solid angles are carried out using numerical quadratures.

The $(d, p n)$ elastic breakup data have been measured at the RIKEN Accelerator Research Facility, Saitama, at 140 and $270 \mathrm{MeV}[9,10]$, and at the Research Center for Nuclear Physics (RCNP), Osaka, at $56 \mathrm{MeV}$ [11], in a kinematical condition of $\theta_{p} \approx \theta_{n} \approx 0^{\circ}$. The targets were ${ }^{12} \mathrm{C},{ }^{28} \mathrm{Si},{ }^{40} \mathrm{Ca}$, ${ }^{90} \mathrm{Zr},{ }^{118} \mathrm{Sn},{ }^{165} \mathrm{Ho}$, and ${ }^{208} \mathrm{~Pb}$ at $E_{d}=140$ and $270 \mathrm{MeV}$ and
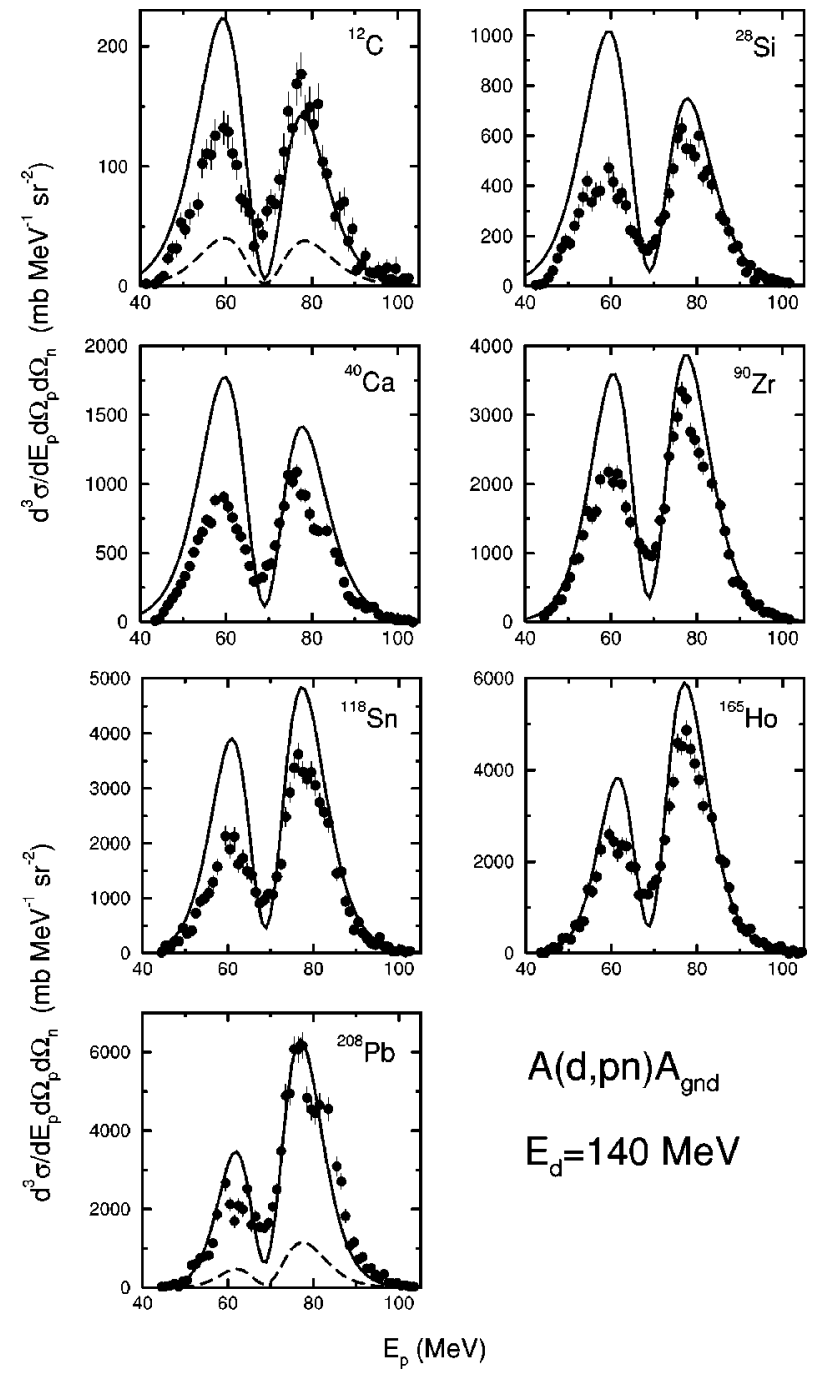

FIG. 3. Experimental and calculated adiabatic model (solid curves) triple differential cross sections for deuteron breakup near $0^{\circ}$ in the laboratory frame at $E_{d}=140 \mathrm{MeV}$. The calculations are averaged over the proton and neutron detection solid angles specified in the text. The dashed curves, for ${ }^{12} \mathrm{C}$ and ${ }^{208} \mathrm{~Pb}$, use the Baur-Trautmann approximation of Eq. (27).

${ }^{12} \mathrm{C},{ }^{40} \mathrm{Ca},{ }^{90} \mathrm{Zr}$, and ${ }^{208} \mathrm{~Pb}$ at $E_{d}=56 \mathrm{MeV}$. The solid angles subtended by the neutron and proton counters will be seen to be of considerable importance. These were $\Delta \Omega_{n}=0.45 \mathrm{msr}$ (with a circular geometry) at $140 \mathrm{MeV}$ and $0.48 \mathrm{msr}$ (with square geometry) at $270 \mathrm{MeV}$. The $\Delta \Omega_{p}=7.2 \mathrm{msr}$ (a 60 $\times 120 \mathrm{mrad}^{2}$ rectangular geometry) at both 140 and 270 $\mathrm{MeV}$. For the $56 \mathrm{MeV}$ data, then $\Delta \Omega_{n}=0.88 \mathrm{msr}$ (with circular geometry) and $\Delta \Omega_{p}=5.6 \mathrm{msr}$ (a $75 \times 75 \mathrm{mrad}^{2}$ square geometry). In all cases the solid angle elements are centered about $\theta_{p}=\theta_{n}=0^{\circ}$.

\section{A. Calculations at $140 \mathrm{MeV}$}

The calculations and data at $140 \mathrm{MeV}[9,10]$ are compared in Fig. 3 for all measured targets. The errors shown in the figure are statistical only. The solid lines show the elastic breakup cross sections, as a function of the detected (laboratory) proton energy, calculated using the finite-range amplitude of Eq. (21) and Eqs. (28), (29), and (30). The overall 


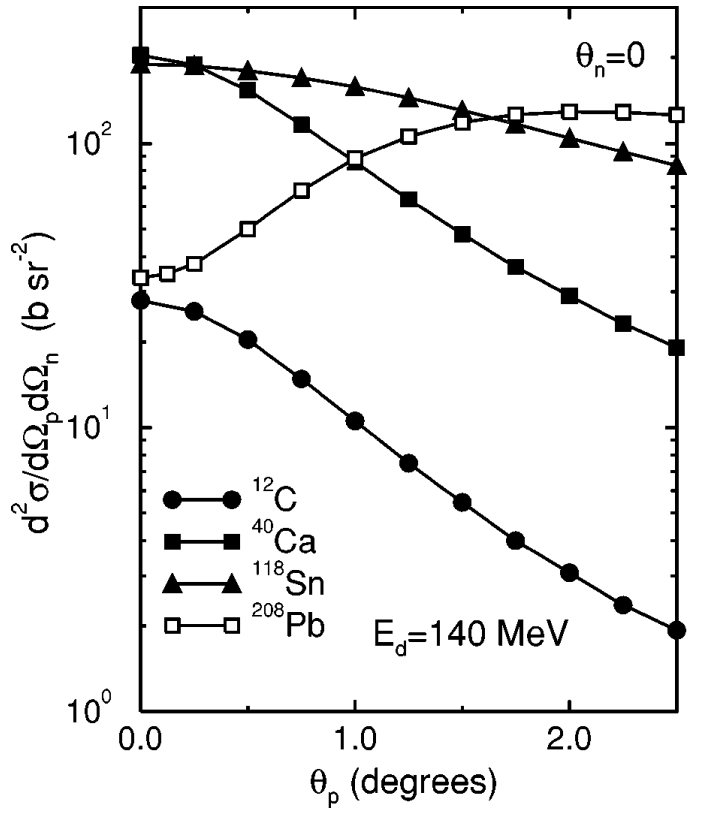

FIG. 4. Calculated double differential cross section angular distributions versus $\theta_{p}\left(\right.$ at $\left.\theta_{n}=0^{\circ}\right)$, integrated over the detected proton energy and without solid angle averaging, for ${ }^{12} \mathrm{C},{ }^{40} \mathrm{Ca},{ }^{118} \mathrm{Sn}$, and ${ }^{208} \mathrm{~Pb}$ at $E_{d}=140 \mathrm{MeV}$.

agreement of the calculated magnitudes, $Z_{t}$ dependence, and the proton energy dependence, with the data is good and improves with increasing target charge. The factor of 40 increase in the magnitudes of the measured cross sections in going from ${ }^{12} \mathrm{C}$ to ${ }^{208} \mathrm{~Pb}$ is seen to be well reproduced as a function of $Z_{t}$. Figure 3 does not, however, reveal the complex way that the calculated cross sections are built up in the integrations over the experimental solid angle acceptances. These are quite different for the small and large $Z_{t}$ targets.

To clarify these differences, in Fig. 4 we show the calculated double differential cross section angular distributions, against $\theta_{p}\left(\right.$ at $\left.\theta_{n}=0^{\circ}\right)$, integrated over the final proton energy - without solid angle averaging. The curves show the representative ${ }^{12} \mathrm{C},{ }^{40} \mathrm{Ca},{ }^{118} \mathrm{Sn}$, and ${ }^{208} \mathrm{~Pb}$ target cases at $E_{d}=140$. The experimental acceptances for the $140 \mathrm{MeV}$ data involve a very small $\Delta \Omega_{n}=0.45 \mathrm{msr}$ and so setting $\theta_{n}$ $=0^{\circ}$ is representative. The proton (spectrometer) acceptance on the other hand is $\Delta \Omega_{p}=7.2 \mathrm{msr}$ (with the rectangular geometry given above) and includes proton angles as large as $\theta_{p} \approx 3.5^{\circ}$ about zero degrees. Over this angular range the intrinsic cross section for ${ }^{12} \mathrm{C}$, with forward going neutrons, is seen to fall by more than an order of magnitude while that for ${ }^{208} \mathrm{~Pb}$ increases by about a factor of 3 .

Figures 5 and 6 show the calculated triple differential cross sections, with $\theta_{n}=0^{\circ}$, for ${ }^{12} \mathrm{C}$ and ${ }^{208} \mathrm{~Pb}$ at $\theta_{p}=0^{\circ}$ (short dashed curves), $\theta_{p}=1^{\circ}$ (dot-dashed curves), and $\theta_{p}$ $=2^{\circ}$ (long dashed curves), showing again the increasing cross section with $\theta_{p}$ for ${ }^{208} \mathrm{~Pb}$ and the rapidly falling cross section with $\theta_{p}$ for ${ }^{12} \mathrm{C}$. Also shown are the experimental data and the correctly calculated averages over the experimental acceptances (solid curves). These figures show that the calculations, and the level of agreement obtained and presented in Fig. 3, do not arise trivially from a simple scaling of calculated cross sections with $Z_{t}$. We see that the trends with $Z_{t}$ and target mass and the obtained cross section

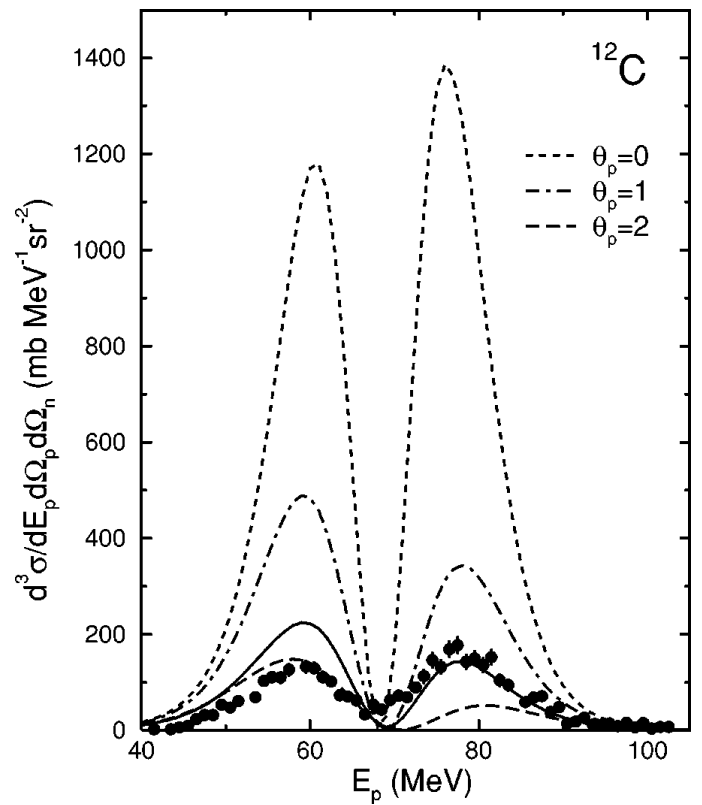

FIG. 5. Calculated triple differential cross section for ${ }^{12} \mathrm{C}$, with $\theta_{n}=0^{\circ}$, at $\theta_{p}=0^{\circ}$ (short dashed curves), $\theta_{p}=1^{\circ}$ (dot-dashed curves), and $\theta_{p}=2^{\circ}$ (long dashed curves) at $E_{d}=140 \mathrm{MeV}$. Also shown are the experimental data and the calculated averages over the experimental acceptances (solid curves).

magnitudes are the result of a careful specification of experimental conditions and of the theory which derives a complicated dependence of the breakup mechanism on target charge and detected proton energy. Evidently the theoretical cross sections calculated at zero degrees have no direct relationship to the data. We return to this point in the following discussion.

Overall, our results are consistent with an underlying physical picture in which Coulomb breakup is the dominant mechanism. There are, however, indications of a missing and interfering contribution, particularly on the lighter targets,

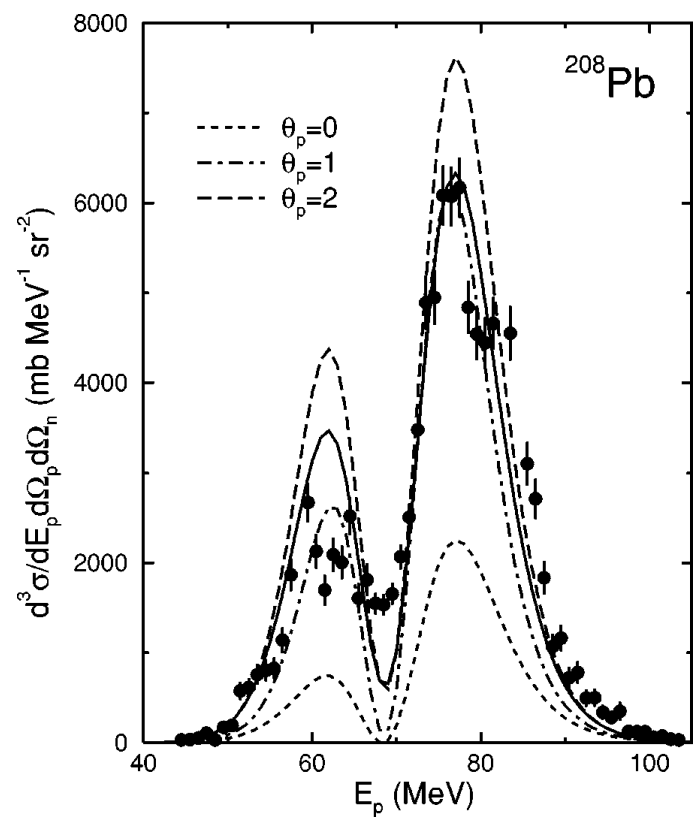

FIG. 6. As for Fig. 5 but for the ${ }^{208} \mathrm{~Pb}$ target at $E_{d}=140 \mathrm{MeV}$. 

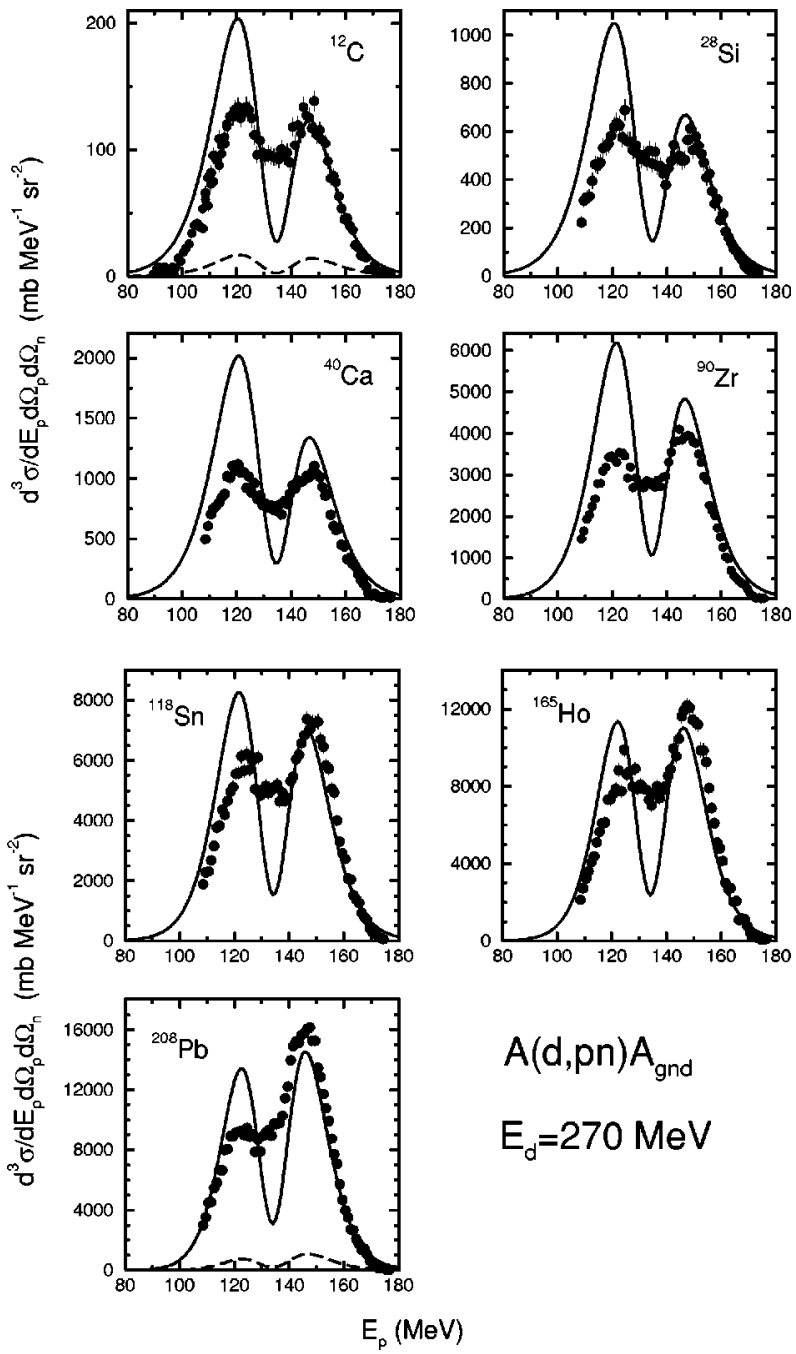

FIG. 7. Experimental and calculated adiabatic model (solid curves) triple differential cross sections for deuteron breakup near $0^{\circ}$ in the laboratory frame at $E_{d}=270 \mathrm{MeV}$. The calculations are averaged over the proton and neutron detection solid angles specified in the text. The dashed curves, for ${ }^{12} \mathrm{C}$ and ${ }^{208} \mathrm{~Pb}$, use the Baur-Trautmann approximation of Eq. (27).

most probably resulting from smaller nuclear breakup contributions.

We should note that if, instead of using the adiabatic theory amplitude of Eq. (21), we use the Baur-Trautmann approximation of Eq. (27) then we calculate the results shown by the long dashed curves in Fig. 3 for ${ }^{12} \mathrm{C}$ and ${ }^{208} \mathrm{~Pb}$. As discussed earlier, the factor $\left\langle\mathbf{q}_{v}\left|V_{v c}\right| \phi_{0}\right\rangle /\left\langle\mathbf{P}_{v}\left|V_{v c}\right| \phi_{0}\right\rangle$ between these two amplitudes results in a considerable underestimation of the data at $140 \mathrm{MeV}$. Since the contributing $\mathbf{q}_{v}$ increase with $E_{d}$ we shall see that this discrepancy is even more serious at $270 \mathrm{MeV}$.

\section{B. Calculations at $270 \mathrm{MeV}$}

The calculations and data at $270 \mathrm{MeV}[10,9]$ are compared in Fig. 7 for all measured targets, the errors shown being statistical only. The solid lines show the solid angle averaged elastic breakup cross sections using Eq. (21). As for the $140 \mathrm{MeV}$ data, the overall agreement of the calculated magnitudes and $Z_{t}$ dependence, with the data is good

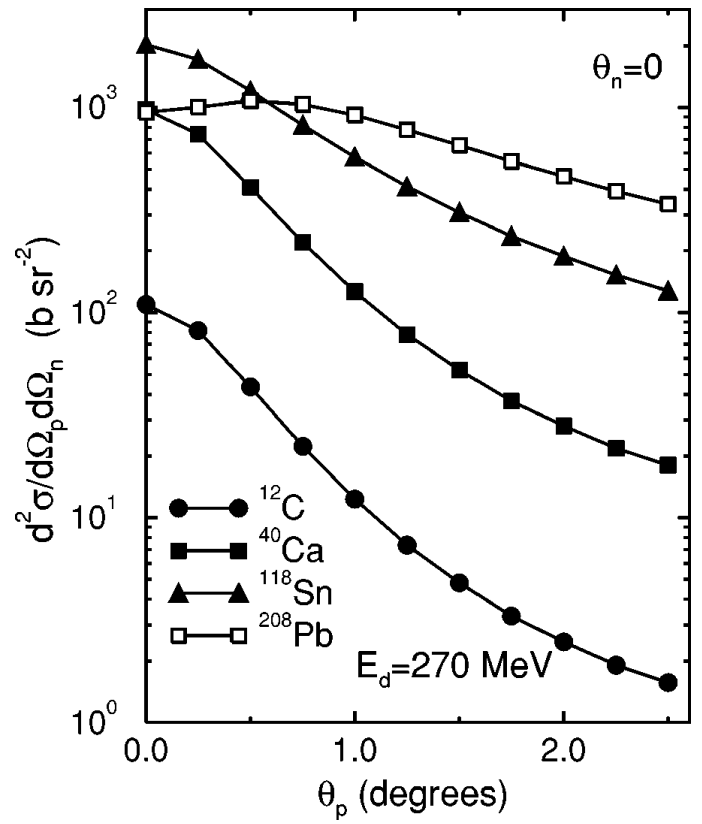

FIG. 8. Calculated double differential cross section angular distributions versus $\theta_{p}\left(\right.$ at $\left.\theta_{n}=0^{\circ}\right)$, integrated over the detected proton energy and without solid angle averaging, for ${ }^{12} \mathrm{C},{ }^{40} \mathrm{Ca},{ }^{118} \mathrm{Sn}$, and ${ }^{208} \mathrm{~Pb}$ at $E_{d}=270 \mathrm{MeV}$.

and improves with increasing target charge. The increasing magnitudes of the measured cross sections, now by a factor of $\approx 160$, in going from ${ }^{12} \mathrm{C}$ to ${ }^{208} \mathrm{~Pb}$ is seen to be well reproduced as a function of $Z_{t}$. Again our results are consistent with a dominant Coulomb breakup mechanism with indications of a missing interference from smaller nuclear contributions. The minima in the calculated cross sections, due to the dominant dipole breakup mechanism, are also filled within the data at $270 \mathrm{MeV}$, additional evidence of a missing nuclear breakup contribution.

The cross sections of Fig. 7, similar to those of Fig. 3, are built up from the integrations (and then the average) over the experimental solid angle acceptances. Figure 8 shows the calculated double differential cross section angular distributions at $270 \mathrm{MeV}$, analogous to Fig. 4, and without solid angle averaging. The experimental acceptances at $270 \mathrm{MeV}$ are essentially the same as at $140 \mathrm{MeV}$ and so similar conclusions apply. Over the proton (spectrometer) acceptance the intrinsic cross section for ${ }^{12} \mathrm{C}$, with forward going neutrons, is now seen to fall by two orders of magnitude while, at $270 \mathrm{MeV}$, that for ${ }^{208} \mathrm{~Pb}$ now also falls slowly with increasing $\theta_{p}$. Once again it is evident that the trends with $Z_{t}$, and the calculated cross section magnitudes, result from a complicated interplay of the theoretical dependences and the experimental kinematical conditions and solid angles involved. The dependences of the Coulomb breakup mechanism, on the incident deuteron energy, target charge, and detected proton energy, within the present theory appear rather consistent with the experimental data. Using the BaurTrautmann approximation of Eq. (27) we calculate the long dashed curves in Fig. 7, for ${ }^{12} \mathrm{C}$ and ${ }^{208} \mathrm{~Pb}$, resulting in a considerable underestimation of the data.

\section{Calculations at $56 \mathrm{MeV}$}

An incident deuteron energy of $56 \mathrm{MeV}$ is at the lower end of the range of energies at which an adiabatic treatment 


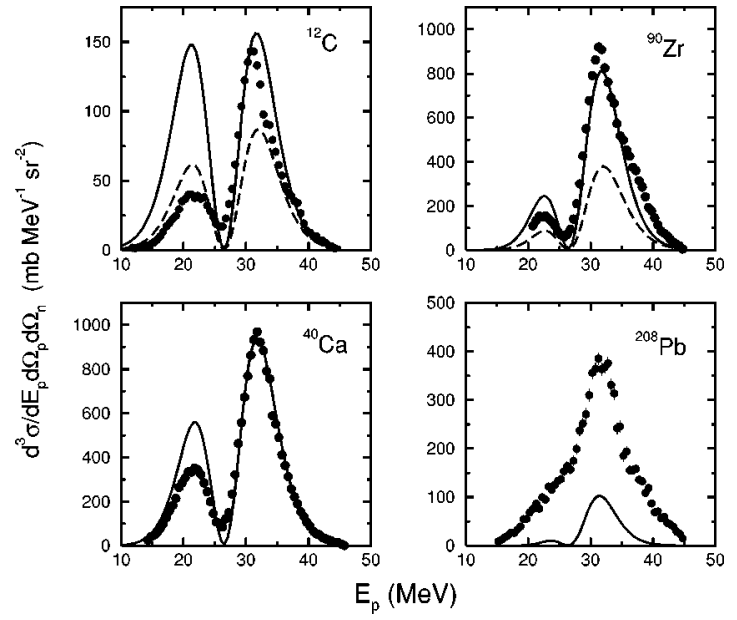

FIG. 9. Experimental and calculated adiabatic model (solid curves) triple differential cross sections for deuteron breakup near $0^{\circ}$ in the laboratory frame at $E_{d}=56 \mathrm{MeV}$. The calculations are averaged over the proton and neutron detection solid angles specified in the text. The dashed curves for ${ }^{12} \mathrm{C}$ and ${ }^{90} \mathrm{Zr}$, use the BaurTrautmann approximation of Eq. (27).

is thought to be reliable, based upon earlier comparisons of adiabatic and nonadiabatic methods for transfer reaction and nuclear breakup processes [26,27]. We include an analysis of these experimental data here both for completeness and since these data were also the subject of an earlier (prior form) DWBA analysis by Samanta et al. [28].

The calculations and data at $56 \mathrm{MeV}$ [11] are compared in Fig. 9 for the four measured targets ${ }^{12} \mathrm{C},{ }^{40} \mathrm{Ca},{ }^{90} \mathrm{Zr}$, and ${ }^{208} \mathrm{~Pb}$. The errors shown are statistical only. The solid lines show the solid angle averaged elastic breakup cross sections calculated using Eq. (21). When using instead the BaurTrautmann approximation to the amplitude, Eq. (27), we calculate the long dashed curves in Fig. 9, for the ${ }^{12} \mathrm{C}$ and ${ }^{90} \mathrm{Zr}$ targets. Since now the $\left|\mathbf{q}_{v}\right|$ involved are smaller, the underestimation of the data is less severe, but it is clear that this model does not include the essential physics correctly at this energy also. We see that the quality of the agreement of the adiabatic model calculations with the data for the three lighter targets is comparable to that for the higher-energy data. The calculations for the ${ }^{208} \mathrm{~Pb}$ target on the other hand underestimate the data rather seriously (about a factor of 4). The origin of this particular discrepancy for ${ }^{208} \mathrm{~Pb}$ is unclear based on the present analysis. The discrepancy might reflect a more significant nuclear breakup contribution, due to the increasing strength of the real distortion and increased surface absorption in the nucleon-target interactions, as the deuteron incident energy is reduced. Alternatively the results might indicate the onset of significant nonadiabatic corrections at this lowest energy.

The calculated double differential cross section angular distributions at $56 \mathrm{MeV}$, analogous to Fig. 8, and without solid angle averaging, are shown in Fig. 10. The angular variations of the cross section, with emerging proton angle, are now much less pronounced, but still forward angle peaked, for the light targets. That for ${ }^{90} \mathrm{Zr}$ is now almost constant. The cross section for ${ }^{208} \mathrm{~Pb}$ is seen to increase rapidly with $\theta_{p}$ and hence, when combined with the $\sin \theta_{p}$ weighting in the $\Delta \Omega_{p}$ solid angle integral, most of the cal-

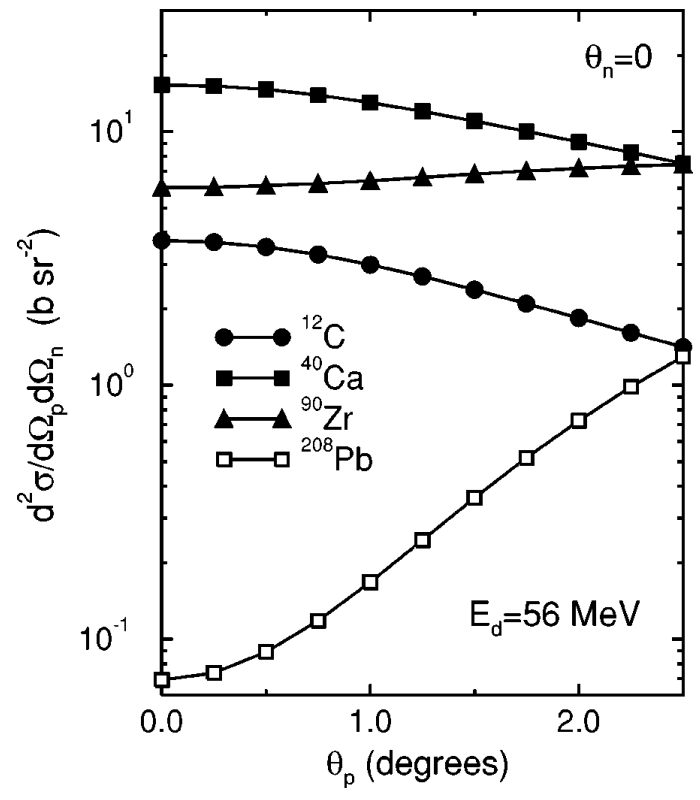

FIG. 10. Calculated double differential cross section angular distributions versus $\theta_{p}\left(\right.$ at $\left.\theta_{n}=0^{\circ}\right)$, integrated over the detected proton energy and without solid angle averaging, for ${ }^{12} \mathrm{C},{ }^{40} \mathrm{Ca}$, ${ }^{90} \mathrm{Zr}$, and ${ }^{208} \mathrm{~Pb}$ at $E_{d}=56 \mathrm{MeV}$.

culated cross section for ${ }^{208} \mathrm{~Pb}$ in Fig. 9 results from the protons emerging at the very largest extremes of the experimental detection solid angles.

Our Coulomb breakup results, which reproduce the magnitudes of the ${ }^{12} \mathrm{C},{ }^{40} \mathrm{Ca}$, and ${ }^{90} \mathrm{Zr}$ data are in contrast to those of the (prior form) DWBA analysis of these same $(d, p n)$ data by Samanta et al. [28]. They reported Coulomb breakup cross sections which significantly overpredicted these data. They also concluded that nuclear breakup contributions are large. Since we have not considered nuclear breakup here, we are unable to comment on this latter feature. Regarding the Coulomb breakup calculations, however, it is not at all clear that the necessary averages of the calculated triple differential cross sections, over the experimental acceptances about zero degrees, were carried out in Ref. [28]. Figure 11 shows, in addition to the angle averaged calculations (solid curves) and data, the triple cross sections calculated strictly at $\theta_{p}=\theta_{n}=0^{\circ}$ (dashed curves). Without the solid angle averaging one would indeed conclude that the Coulomb dissociation contribution is too large on the light, ${ }^{12} \mathrm{C}$ and ${ }^{40} \mathrm{Ca}$, targets.

\section{General features}

We have shown that, even in the presence of only pure Coulomb breakup, the angular distributions of the dissociated fragments have a complex dependence on the incident projectile energy and the target mass and charge. In particular, there is no simple scaling of the calculations with the target charge. We summarize this information in Fig. 12 which shows the calculated double differential cross sections at and near zero degrees as a function of the target nucleus charge, integrated over the detected proton energy. The circle, square, and diamond symbols show the results at $E_{d}=140,270$, and $56 \mathrm{MeV}$, respectively. The solid symbols are the calculations which have been averaged over the pro- 


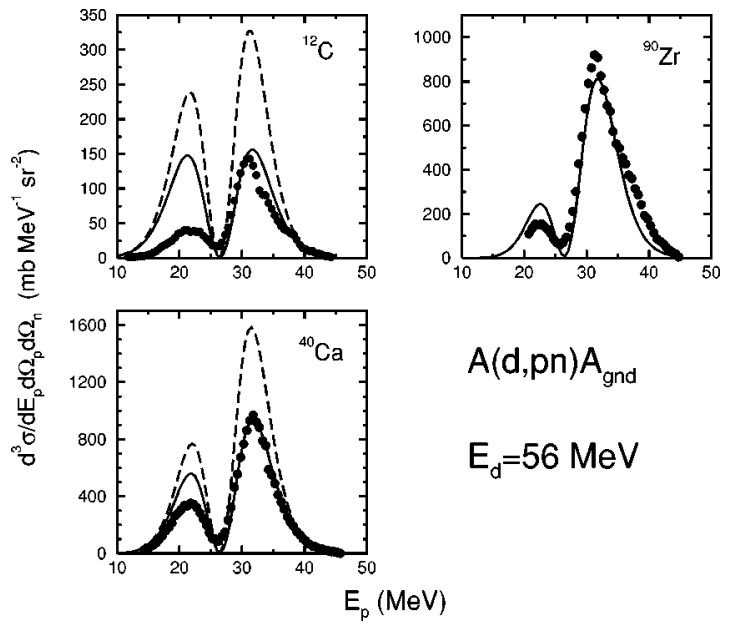

FIG. 11. Experimental and calculated adiabatic model (solid curves) triple differential cross sections for deuteron breakup near $0^{\circ}$ in the laboratory frame at $E_{d}=56 \mathrm{MeV}$. The calculations are averaged over the proton and neutron detection solid angles specified in the text. The dashed curves are the cross sections calculated strictly at $\theta_{p}=\theta_{n}=0^{\circ}$.

ton and neutron detection solid angles. The open symbols are the cross sections calculated strictly at $\theta_{p}=\theta_{n}=0^{\circ}$. Several general trends are evident.

With reference to the open symbols, the cross sections calculated strictly at $\theta_{p}=\theta_{n}=0^{\circ}$ show a characteristic trend with increasing energy. All calculations show a maximum at a given $Z_{t}$, whose value, and the magnitude of the cross section, increases with incident energy. At all energies the

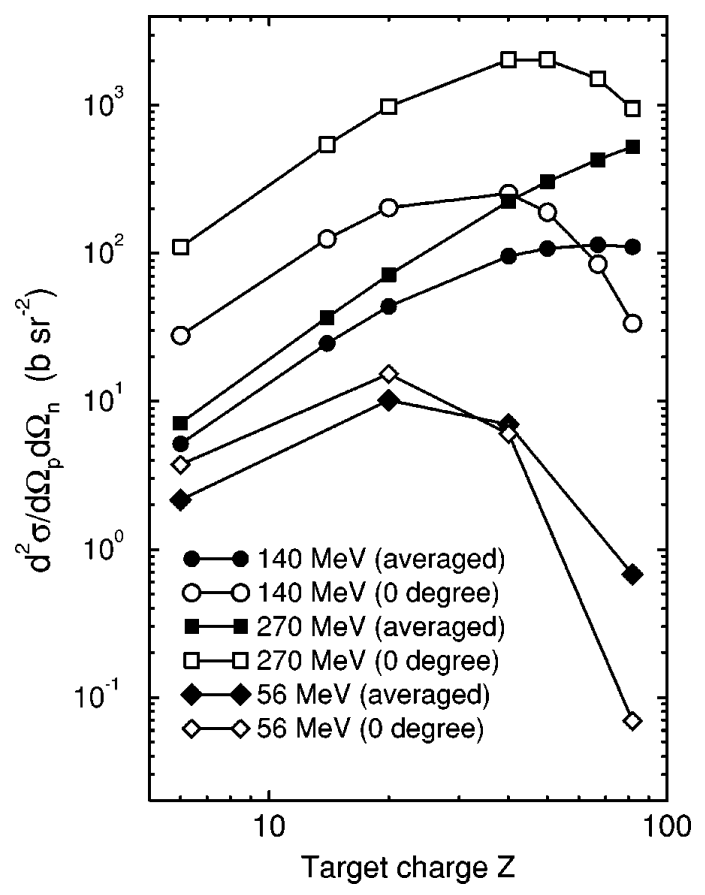

FIG. 12. Calculated double differential cross sections at zero degrees, versus the target nucleus charge, integrated over the detected proton energy, at $E_{d}=140,270$, and $56 \mathrm{MeV}$. The solid symbols show the calculations when averaged over the proton and neutron detection solid angles. The open symbols are the cross sections calculated strictly at $\theta_{p}=\theta_{n}=0^{\circ}$. $\theta_{p}=\theta_{n}=0^{\circ}$ differential cross section is smaller for ${ }^{208} \mathrm{~Pb}$ than for several of the lighter nuclei. For the lower two energies, 140 and $56 \mathrm{MeV}$, the ${ }^{208} \mathrm{~Pb}$ cross section at $\theta_{p}=\theta_{n}$ $=0^{\circ}$ is smaller or comparable to that for ${ }^{12} \mathrm{C}$.

The calculated angular distributions of the core (proton) fragment, for $\theta_{n}=0^{\circ}$, shown in Figs. 4, 8, and 10, mean that, when integrated over the small experimental solid angle acceptances, the picture is very different, as shown by the solid symbols in Fig. 12. The almost straight line (on the log-log plot) for the calculated cross sections at $270 \mathrm{MeV}$, indicative of a simple power law dependence on $Z_{t}$, is therefore entirely the result of the particular chosen experimental detector arrangement. The information within the measurements is much more complex and further investigations, using different solid angle geometries would be very interesting. In all cases, however, the forward peaked proton angular distributions for the lightest targets means that the measurements are considerably reduced compared to the $\theta_{p}=\theta_{n}=0^{\circ}$ results. The opposite is true for the most massive targets where the finite solid angles increase the measured effective cross sections.

We conclude by noting that the present method, based on the solution of Eq. (1), depends critically on the approximation that only one projectile constituent effectively interacts with the target. The method is not immediately applicable therefore either to the treatment of breakup in cases where the projectile has two loosely bound and charged constituents, or to the treatment of breakup due to the nuclear components of the core and valence particle-target interactions $V_{c t}^{N}$ and $V_{v t}^{N}$. This is particularly the case for the deuteron where, for the two nucleons $V_{c t}^{N} \approx V_{v t}^{N}$. The method could nevertheless provide a good first approximation for systems, such as halo nuclei, with a large core to valence particle mass ratio, or where $V_{c t} \gg V_{v t}$, e.g., Ref. [13].

\section{SUMMARY AND CONCLUSIONS}

We have considered the Coulomb dissociation of a composite projectile comprising a charged core and a neutral valence particle. We have shown that the use of a single approximation, that breakup is to the low-energy continuum, leads to a simple and transparent expression for the post form breakup amplitude in the limit that strong interaction effects are neglected. The treatment includes a fully finiterange treatment of the valence-core particle interaction $V_{v c}$ and does not make the weak coupling approximation of the DWBA.

We have compared the parameter free theoretical predictions with high precision differential deuteron dissociation measurements near zero degrees, at incident deuteron energies of 140, 270, and $56 \mathrm{MeV}$. We obtain a good agreement with experiment over the full range of measured targets, from $Z_{t}=6$ to 82 , at 140 and $270 \mathrm{MeV}$. We obtain a similar agreement with the data for ${ }^{12} \mathrm{C},{ }^{40} \mathrm{Ca}$, and ${ }^{90} \mathrm{Zr}$ targets at 56 $\mathrm{MeV}$, but we underestimate the data for ${ }^{208} \mathrm{~Pb}$ at this lowest energy.

We have argued that the zero-range approximation to the finite-range DWBA breakup amplitude is very suspect at the energies of interest here. It cannot be justified either in the 
present light-ion context or for related halo systems with similar incident energies/nucleon. We have shown, however, that if applied, then the zero-range approximation leads to an expression which, fortuitously, is equal to the $\mathbf{P}_{v}=0$ approximation to our finite-range adiabatic breakup amplitude. We believe therefore that the physical picture suggested by the zero-range DWBA, regarding the importance of finiterange and multistep effects, is quite misleading and that the amplitudes actually calculated should instead be viewed as a good approximation to a finite-range, nonperturbative, adiabatic breakup amplitude.

\section{ACKNOWLEDGMENTS}

The financial support of the United Kingdom Engineering and Physical Sciences Research Council (EPSRC) in the form of Grant No. GR/J95867, and of the Thai Government (for S.R.) is gratefully acknowledged. The authors would like to express their thanks to Dr. H. Okamura, RIKEN/ University of Tokyo for providing the experimental data used here in tabular form and for his detailed specification of the experimental conditions. Travel support, in the early stages of this work, from the British Council is also gratefully acknowledged.
[1] A. C. Shotter, V. Rapp, T. Davinson, D. Branford, N. E. Sanderson, and M. A. Nagarajan, Phys. Rev. Lett. 53, 1539 (1984).

[2] G. Baur and H. Rebel, J. Phys. G 20, 1 (1994).

[3] I. Tanihata et al., Phys. Lett. 160B, 380 (1985); Phys. Rev. Lett. 55, 2676 (1985).

[4] C. A. Bertulani and F. Canto, Nucl. Phys. A539, 163 (1992).

[5] H. Esbensen, G. F. Bertsch, and C. A. Bertulani, Nucl. Phys. A581, 107 (1995); T. Kido, K. Yabana, and Y. Suzuki, Phys. Rev. C 53, 2296 (1996), and references therein.

[6] S. Typel and G. Baur, Nucl. Phys. A573, 486 (1994).

[7] L. F. Canto, R. Donangelo, A. Romanelli, M. S. Hussein, and A. F. R. de Toledo Piza, Phys. Rev. C 55, R570 (1997).

[8] S. Typel and G. Baur, Phys. Rev. C 50, 2104 (1994); H. Esbensen, and G. F. Bertsch, Nucl. Phys. A600, 37 (1996); S. Typel, H. H. Wolter, and G. Baur, ibid. A613, 147 (1997).

[9] J. A. Tostevin et al., Phys. Lett. B (to be published).

[10] H. Okamura, S. Ishida, N. Sakamoto, H. Otsu, T. Uesaka, T. Wakasa, H. Sakai, T. Niizeki, H. Toyokawa, Y. Tajima, H. Ohnuma, M. Yosoi, K. Hatanaka, and T. Ichihara (in preparation).

[11] H. Okamura, S. Hatori, N. Matsuoka, T. Noro, A. Okihana, H. Sakai, H. M. Shimizu, K. Takeshita, and T. Yamaya, Phys. Lett. B 325, 308 (1994).

[12] R. C. Johnson and P. J. R. Soper, Phys. Rev. C 1, 976 (1970).

[13] R. C. Johnson, J. S. Al-Khalili, and J. A. Tostevin, Phys. Rev. Lett. 79, 2771 (1997).
[14] R. C. Johnson, Elastic Scattering and Elastic Break-up of Halo Nuclei in a Special Model, Proceedings of the European Conference on Advances in Nuclear Physics and Related Areas, Thessaloniki, Greece, 1997 (unpublished).

[15] J. A. Tostevin, R. C. Johnson, and J. S. Al-Khalili, Nucl. Phys. A630, 340c (1998).

[16] F. J. Rybicki, PhD thesis, University of Pittsburgh 1969, Appendix A.

[17] N. Austern, Direct Nuclear Reaction Theories (Wiley, New York, 1970), p. 13.

[18] G. Baur and D. Tratumann, Nucl. Phys. A191, 321 (1972).

[19] A. Nordsieck, Phys. Rev. 93, 785 (1954).

[20] P. Banerjee and R. Shyam, Nucl. Phys. A561, 112 (1993).

[21] R. Shyam, P. Banerjee, and G. Baur, Nucl. Phys. A540, 341 (1992).

[22] L. Hulthén and M. Sugawara, in Handbuch der Physik, edited by S. Flügge (Springer, Berlin, 1957), Vol. XXXIX, p. 92.

[23] R. V. Reid, Jr., Ann. Phys. (N.Y.) 50, 441 (1968).

[24] G. G. Ohlson, Nucl. Instrum. Methods 37, 240 (1965).

[25] H. Fuchs, Nucl. Instrum. Methods Phys. Res. 200, 361 (1982).

[26] M. Kamimura, M. Yahiro, Y. Iseri, H. Kameyama, Y. Sakuragi, and M. Kawai, Prog. Theor. Phys. Suppl. 89, 1 (1986).

[27] N. Austern, Y. Iseri, M. Kamimura, M. Kawai, G. Rawitscher, and M. Yahiro, Phys. Rep. 154, 125 (1987).

[28] C. Samanta, S. Mukherjee, R. Kanungo, and D. N. Basu, Phys. Rev. C 53, 2287 (1996). 